\title{
Analysis and fragmentation of organic samples by (low-energy) dynamic SIMS
}

\author{
K. Q. Ngo, ${ }^{a *}$ P. Philipp, ${ }^{a}{ }^{\text {Y. Jin, }}{ }^{\text {b }}$ S. E. Morris, ${ }^{c}$ M. Shtein, ${ }^{\text {b }}$ J. Kieffer ${ }^{b}$ \\ and T. Wirtz
}

Up to now, the analysis of organic or biological samples was mainly investigated using static SIMS, while dynamic SIMS was generally limited to the analysis of inorganic samples. The increasing sophistication of organic optoelectronic devices (e.g. organic light emitting diodes and organic photovoltaic cells, etc.) requires molecular-level dimensional control in the fabrication of multilayered structures with specifically engineered interfaces. However, analytical tools for monitoring such fabrication precision are scarce. In a current project, we address this challenge by advancing the development of low-energy Secondary Ion Mass Spectrometry (LE-SIMS) for the analysis of organic-based optoelectronic materials systems.

In the present work, we investigate the fragmentation as well as the ionization mechanisms for several molecules used in such devices: fullerene, copper phthalocyanine and tris(8-hydroxyquinolinato) aluminium have been deposited onto silicon wafers. The study has been carried out on a Cameca SC-Ultra instrument under $\mathrm{Cs}^{+}$bombardment for various impact energies in the $\mathrm{M}^{-}$mode. Constant $\mathrm{M}^{-}$secondary ion intensities have been observed throughout the organic layers for some characteristic fragments of the organic molecules. Copyright (c) 2010 John Wiley \& Sons, Ltd.

Keywords: SIMS; sputtering; impact energy; fragmentation; cluster abundance distribution

\section{Introduction}

In recent years, organic optoelectronic devices (e.g. organic light emitting diodes and organic photovoltaic cells) have known an increasing interest because of their low fabrication costs. With the increasing sophistication of the devices, a meticulous dimensional control of the layered structures is required. However, due to a lack of adequate characterization tools, the influence of growth parameters on the structure remains largely unverified. In a current project, we use low-energy Secondary lon Mass Spectrometry (LE-SIMS) to enhance our knowledge of the interfacial structure and electronic properties of such devices. When analyzing such samples by depth-profiling in dynamic SIMS, knowledge of the fragmentation of the organic molecules over a large range of primary ion fluency is essential.

Over the last few decades, the fragmentation and cluster formation of matter sputtered under ion bombardment in dynamic SIMS has been investigated in many studies. ${ }^{[1-7]}$ It was found that the abundance of charged clusters fluctuates as a function of their size, while the distribution of the neutral clusters decreases continuously with the cluster size. ${ }^{[1,2]}$ This behavior has been related to the variation of the ionization energy rather than to that of the binding energy. The ejection process was assumed to occur in two steps: sputter emission of a neutral cluster followed by the ionization as it leaves the interaction range with the surface. The intensity oscillations in the mass spectra of cluster ions are, therefore, an artefact caused by ionization of the neutral clusters. ${ }^{[2]}$ In the case of carbon clusters sputtered from graphite, a correlation between the abundance distribution and the electron affinity was confirmed. ${ }^{[4-6]}$ Results in literature are mainly limited to metals, ${ }^{[1-3,8]}$ alloys and polycrystalline or amorphous inorganic materials, ${ }^{[4-7,9-12]}$ but no studies on organic molecules have been noted as of yet.
In this paper, we study the fragmentation of $\mathrm{C}_{60}$, copper phthalocyanine (CuPC) and aluminium tris(8-hydroxyquinoline) $\left(\mathrm{Alq}_{3}\right)$ molecules under (low energy) bombardment. To our knowledge, this is the first time that the influence of the impact energy on the fragmentation mechanisms of this kind of molecules has been investigated. The alternations in the abundance distribution of $\mathrm{C}_{n}{ }^{-}$and $\mathrm{C}_{n} \mathrm{Cs}^{-}$clusters have been reported and investigated previously, but only at high-impact energies (at $14.5 \mathrm{keV}^{[4-6,10-12]}$ and $5 \mathrm{keV}^{[7]} \mathrm{Cs}^{+}$ion bombardment, at $4 \mathrm{keV} \mathrm{SF}_{5}{ }^{+}, \mathrm{Xe}^{+}$and $\mathrm{Ar}^{+}$ion bombardment ${ }^{[9]}$ ) on different allotropes of carbon, and no results have been published for $\mathrm{C}_{n} \mathrm{~N}_{X}{ }^{-}$clusters sputtered from nitrogen-containing samples.

\section{Experimental}

The fragmentation of the organic molecules was studied on the Cameca SC-Ultra ${ }^{[13]}$ in the negative mode for impact energies between $250 \mathrm{eV}$ and $5 \mathrm{keV}$ and primary ion currents ranging between 1.5 and $30 \mathrm{nA}$. The mass resolution $\frac{M}{\Delta M}$ was set to 400 . A contrast aperture of $300 \mu \mathrm{m}$ was used. The analyzed area was

\footnotetext{
* Correspondence to: K. Q. Ngo, Department "Science and Analysis of Materials" (SAM), Centre de Recherche Public-Gabriel Lippmann, 41 rue du Brill, L-4422 Belvaux, Luxembourg.E-mail:ngo@lippmann.lu

a Department "Science and Analysis of Materials" (SAM), Centre de Recherche Public - Gabriel Lippmann, 41 rue du Brill, L-4422 Belvaux, Luxembourg

b Department of Materials Science and Engineering, University of Michigan, Ann Arbor, Michigan 48109-2136, USA

c Department of Chemical Engineering, University of Michigan, Ann Arbor, Michigan 48109-2136, USA
} 


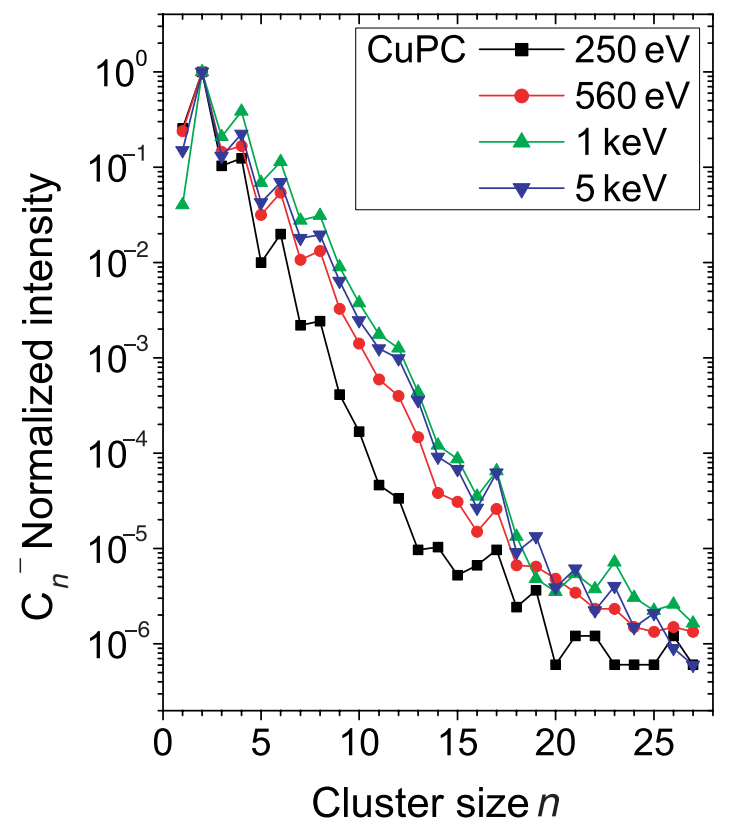

Figure 1. Abundance of $\mathrm{C}_{n}{ }^{-}$clusters as a function of the cluster size $n$ for different impact energies, sputtered from a CuPC film on Si (normalized with respect to $C_{2^{-}}$intensities).

limited to a diameter of $100 \mu \mathrm{m}$ (field aperture of $1200 \mu \mathrm{m}$ ) and the energy slit was closed to a width of $45 \mathrm{eV}$. The primary ion beam was raster-scanned over an area of $500 \mu \mathrm{m} \times 500 \mu \mathrm{m}$. For insulating samples (CuPC and $\mathrm{Alq}_{3}$ ), the electron gun was used for charge compensation.

Thin layers of $C_{60}(43 \mathrm{~nm}), \mathrm{Alq}_{3}(100 \mathrm{~nm})$ and CuPC $(150 \mathrm{~nm})$ were deposited onto silicon wafers using conventional vacuum thermal evaporation at $10^{-6}$ Torr onto substrates at room temperature. During the deposition process, thicknesses were controlled using a precalibrated quartz crystal monitor. $\mathrm{Alq}_{3}$ has aluminium bonded to three 8-hydroxyquinoline groups $\left(\mathrm{C}_{9} \mathrm{H}_{7} \mathrm{NO}\right)$ and thus includes 5.8 atomic \% of nitrogen. The CuPC molecule is a complex of copper and phthalocyanine, where the copper is at the centre of the phthalocyanine. It contains 14 atomic \% of nitrogen.

\section{Results and Discussion}

For each sample, a mass spectrum is taken at different impact energies to study the fragmentation mechanisms with respect to the impact energy. The secondary ion intensities have been recorded between $11 \mathrm{amu}$ and $325 \mathrm{amu}$ under steady-state conditions.

Figure 1 shows the secondary ion intensities of the singly charged $\mathrm{C}_{n}{ }^{-}$clusters sputtered from CuPC as a function of the cluster size for different impact energies. The mass spectra at different impact energies were made with the same sample potential $(2 \mathrm{kV})$, but as primary current and sputter yield change, and some minor variations in the adjustment of the electron gun are possible, the raw data is difficult to compare. Thus, for better comparison, the results were normalized with respect to the highest intensity of each distribution ( $\mathrm{C}_{2}{ }^{-}$ions). On $\mathrm{C}_{60}$ and $\mathrm{Alq}_{3}$, similar results have been obtained (they are not shown due to a lack of space). For the small clusters, an oscillation of the cluster abundance is observed, with the even-numbered clusters being more abundant than the odd-numbered clusters. Around $n=9$, a change in the periodicity occurs: the odd-even oscillations become less pronounced and above $n=16$ the odd-numbered clusters dominate. Such behavior has been observed previously on graphite ${ }^{[4,6]}$ and silicon carbide. ${ }^{[1]}$ Those observations have been attributed to a change in the cluster geometry, which reflects also the variations of the electron affinity. ${ }^{[4,6,11]}$ Ultraviolet photoelectron spectra (UPS) show that small clusters $(n \leq 9)$ have linear chain-like structures, whereas larger ones form monocyclic rings. ${ }^{[5]}$ The correlation between the $C_{n}{ }^{-}$distribution and the electron affinity (the even-odd alternations and the change in distribution from $n=9$ to $n=16$ ) supports the electron tunneling model developed for metals ${ }^{[3]}$ where the secondary ion intensities of stable negatively charged clusters are determined by the electron affinity. ${ }^{[4-6,11]}$ For clusters of similar size, the differences in binding energy are small. ${ }^{[6]}$

For $250 \mathrm{eV}$, a primary ion current smaller than $15 \mathrm{nA}$ and a reduced transmission of the mass spectrometer (extraction voltage of $2 \mathrm{kV}$ ) result in relatively low secondary ion intensities so that intensities of clusters with $n>20$ are severely affected by noise. For the other impact energies, up to $n=27$, the cluster intensities are significantly higher and show an improved reproducibility. Thus, for the distributions at $560 \mathrm{eV}, 1$ and $5 \mathrm{keV}$ (Fig. 1), the oscillatory behavior of the large clusters $(n \geq 15)$ gets more pronounced for the higher-impact energies. The variation of the $\mathrm{C}^{-} / \mathrm{C}_{2}{ }^{-}$ratio could be caused by a charging effect at the beginning of the mass spectra, even though the electron gun was used. Moreover, the difference in structure between our three molecules and graphite does not play a significant role in the sputter-formation of the $\mathrm{C}_{n}{ }^{-}$clusters.

Even-odd alternations similar to the $\mathrm{C}_{n}{ }^{-}$ones are also observed for the heteronuclear $\mathrm{C}_{n} \mathrm{Cs}^{-}$clusters (Fig. 2). Here, they are even much more pronounced. This distribution agrees with both the variation of the binding energy of ground-state isomers $\mathrm{C}_{n} \mathrm{Cs}^{-}$ ( $n=1-10)$ clusters and the electron affinity of $C_{n}$ Cs clusters, ${ }^{[14]}$ It has also been calculated that the $\mathrm{C}_{n} \mathrm{Cs}^{-}(n=4-10)$ ground-state structure is composed of $\mathrm{Cs}^{+}$and $\mathrm{C}_{n}{ }^{2-}$ with $\mathrm{Cs}$ slightly embraced by $C_{n}{ }^{-}$For each impact energy, almost identical distributions of $\mathrm{C}_{n} \mathrm{Cs}^{-}$have been obtained for $\mathrm{C}_{60}, \mathrm{Alq}_{3}$ and $\mathrm{CuPC}$, however, they change with increasing impact energy. For the lowest impact energy $(250 \mathrm{eV})$, even-odd oscillations are seen up to $n=13$. The secondary ion intensities are too low to observe larger clusters. The behavior of the first three clusters changes for the $560 \mathrm{eV}$ impact energy: the oscillations start only at $n=3$ and continue until $n=15$, which is the highest-mass cluster observed in our mass range. For the higher-impact energies ( 1 and $5 \mathrm{keV}$ ), the oscillations are less pronounced than for the low-energy bombardment. Furthermore, the oscillatory behavior is observed only for $n>2$ and $n>5$, respectively. Merely the distribution of $\mathrm{C}_{n} \mathrm{Cs}^{-}$at $250 \mathrm{eV}$ is similar to the results obtained on graphite by Gnaser. ${ }^{[4,6]}$ Differences between our and his results are probably related to the different incidence angles on both instruments (Cameca $4 \mathrm{f}$ with primary column at $30^{\circ}$ for Gnaser and Cameca SC-Ultra with primary column at $60^{\circ}$ for this study). Throughout the $\mathrm{C}_{n} \mathrm{Cs}^{-}$distributions, the clusters with an even number of $\mathrm{C}$ atoms are in between one and two orders of magnitude more abundant than their odd-numbered neighbors. The most intense clusters are $\mathrm{C}_{4} \mathrm{Cs}^{-}$and $\mathrm{C}_{6} \mathrm{Cs}^{-}$. Some experiments at higher mass resolution (3000 instead of 400) at $560 \mathrm{eV}$ show that some $\mathrm{C}_{n} \mathrm{Cs}^{-}$ intensities are affected by mass interference coming from $\mathrm{C}_{m} \mathrm{H}^{-}$. Mass interferences are particularly high for $\mathrm{CCs}^{-}$and $\mathrm{C}_{3} \mathrm{Cs}^{-}$. This behavior changes, however, between 560 and $250 \mathrm{eV}$. 


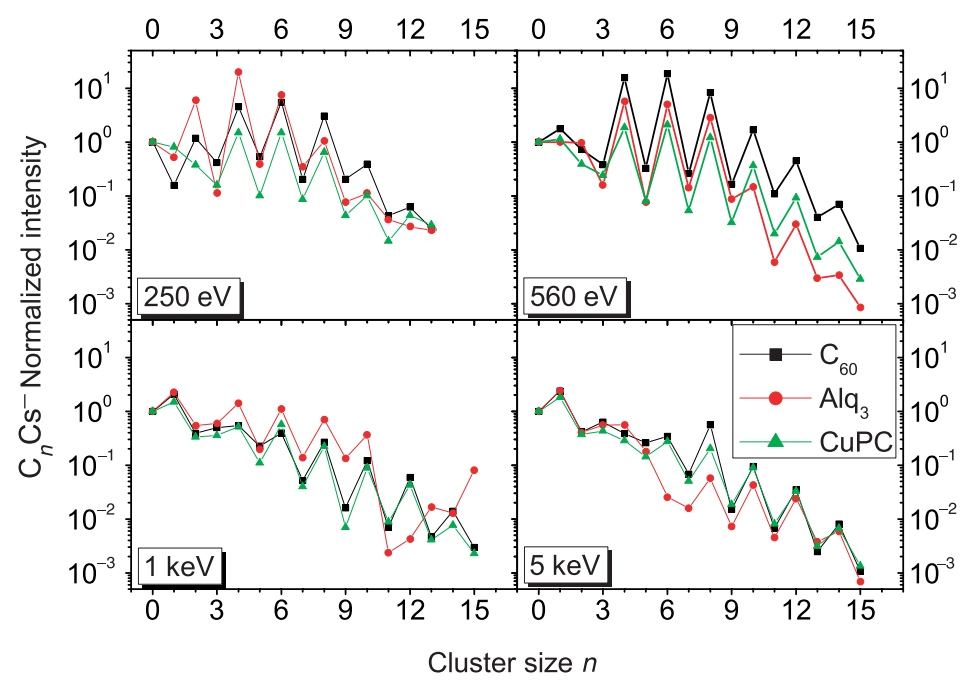

Figure 2. Abundance of $C_{n} C_{s}{ }^{-}$clusters as a function of the cluster size $n$ for different impact energies and sputtered from $C_{60}, A_{l} q_{3}$ and $C u P C$ (normalized with respect to $\mathrm{Cs}^{-}$intensities).

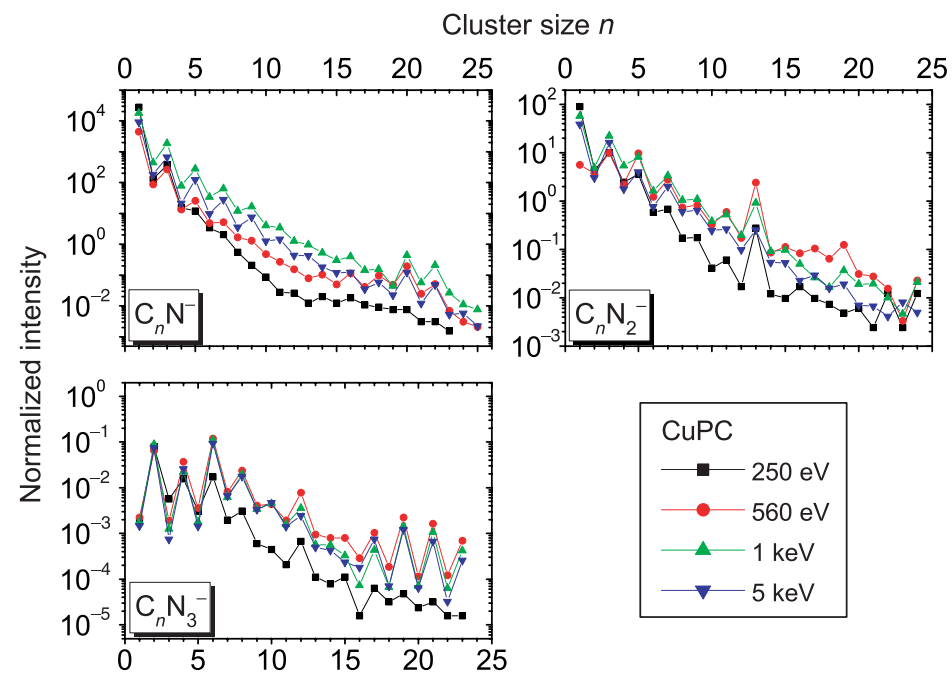

Figure 3. Abundance distribution of the $\mathrm{C}_{n} \mathrm{~N}^{-}, \mathrm{C}_{n} \mathrm{~N}_{2}{ }^{-}$and $\mathrm{C}_{n} \mathrm{~N}_{3}{ }^{-}$clusters as a function of the cluster size $n$ for different impact energies and sputtered from CuPC (normalized with respect to $N^{-}, N_{2}$ and $N_{3}$ intensities, respectively).

For $\mathrm{Alq}_{3}$, the abundance distribution differs for some cluster sizes when compared to $\mathrm{C}_{60}$ and $\mathrm{CuPC}$ at the impact energies of 1 and $5 \mathrm{keV}$. At $1 \mathrm{keV}$, this is the case for $n>11$, while it is for $n=6$ at the $5 \mathrm{keV}$ impact energy. This behavior is probably related to the experimental method: $\mathrm{Alq}_{3}$ being slightly insulating, the electron gun has been used for charge compensation. Indeed, although $\mathrm{C}_{60}, \mathrm{CuPC}$ and $\mathrm{Alq}_{3}$ are used in optoelectronic devices, the electron mobility is lower than for inorganic materials, especially for the latter two.

The distributions of the $\mathrm{C}_{n} \mathrm{~N}^{-}, \mathrm{C}_{n} \mathrm{~N}_{2}{ }^{-}, \mathrm{C}_{n} \mathrm{~N}_{3}$ - clusters are shown for the CuPC sample (Fig. 3). The cluster distributions are similar for the different impact energies. This is in contrast to $\mathrm{Alq}_{3}$ and $\mathrm{C}_{60}$ samples, where distributions vary significantly with energy. Overall, for $\mathrm{C}_{n} \mathrm{~N}^{-}$we found abundance distributions similar to the $\mathrm{C}_{n}{ }^{-}$distributions, except that the clusters with an odd number of $C$ atoms are more abundant than their even-numbered neighbours: these oscillations are more intense for $n \leq 9$ and continue at $n \geq 16$. Our results can be compared to Gupta's experiments ${ }^{[7]}$ where $\mathrm{C}_{60}$ was sputtered in the presence of $\mathrm{N}_{2}$ and $\mathrm{NO}_{2}$. According to this author, the chain-to-ring transition does not occur up to $n=10$. With his experimental setup, he was limited to that size of clusters so that no conclusions can be drawn for the larger clusters observed in our experiments. Furthermore, Zhan's DFT calculations correlate well with our even-odd alternations. ${ }^{[15]}$ Moreover, his $a b$ initio calculations show that the anions $\mathrm{C}_{2} \mathrm{~N}^{-}, \mathrm{C}_{3} \mathrm{~N}^{-}$and $\mathrm{C}_{5} \mathrm{~N}^{-}$ have stable linear structures, while the other $\mathrm{C}_{n} \mathrm{~N}^{-}$anions with larger odd values of $n$ are slightly bent. $C_{n} \mathrm{~N}^{-}$anions with even $n$ (except for $n=2$ ) are bent. For the $\mathrm{C}_{n} \mathrm{~N}_{2}{ }^{-}$distributions of the small cluster sizes, the oscillations in the abundance distributions are similar to the ones of the $\mathrm{C}_{n} \mathrm{~N}^{-}$clusters (maximum intensities for clusters with an odd number of $C$ atoms). Additionally there are two important peaks at $n=13$ and $n=19$. For the $C_{n} N_{3}{ }^{-}$clusters, the situation is changing. Here, the clusters with an even number of $C$ atoms have the highest intensities. The oscillations of $C_{n} N_{3}-$ for both the small $(n \leq 9)$ and the large $(n \geq 16)$ cluster sizes are also more pronounced than those of the $\mathrm{C}_{n} \mathrm{~N}^{-}$and $\mathrm{C}_{n} \mathrm{~N}_{2}{ }^{-}$clusters. Furthermore, the $\mathrm{C}_{12} \mathrm{~N}_{3}{ }^{-}$cluster intensity is much higher than the neighboring intensities. 


\section{Conclusion}

The mechanisms leading to cluster formation in ion bombardment have been thoroughly studied for high-impact energies on inorganic samples. In this paper, we extend the research on cluster formation to organic molecules $\left(\mathrm{C}_{60}, \mathrm{CuPC}\right.$ and $\left.\mathrm{Alq}_{3}\right)$ under various impact energies down to $250 \mathrm{eV}$. The molecules investigated in this study as well as the irradiation conditions are of particular interest for organic optoelectronic devices. The distributions of the $\mathrm{C}_{n}{ }^{-}$and $\mathrm{C}_{n} \mathrm{~N}_{x}{ }^{-}$cluster intensities do not depend on the impact energy, at least not for the energy range between $250 \mathrm{eV}$ and $5 \mathrm{keV}$ investigated in this paper. In contrast to those clusters, the distribution of the $\mathrm{C}_{n} \mathrm{Cs}^{-}$cluster intensities changes with the impact energy: for small impact energies, oscillations are observed over the whole abundance distribution while for impact energies larger than $1 \mathrm{keV}$, the oscillations of the cluster intensities start only at larger cluster sizes. Furthermore, the distributions of the $\mathrm{C}_{n}{ }^{-}$ and $\mathrm{C}_{n} \mathrm{Cs}^{-}$cluster intensities are similar to the results published for graphite, amorphous $\mathrm{C}, \mathrm{C}_{60}$ and $\mathrm{SiC}$ by other authors, indicating that the emission and ionization mechanisms for these different kinds of materials should be similar. For the $C_{n} N_{x}$ - clusters (with $x=1,2,3)$, the alternations in the abundance distributions are only observed for CuPC, but not for $\mathrm{Alq}_{3}$. Thus, the structure of the molecule has an influence on the formation mechanism of the $\mathrm{C}_{n} \mathrm{~N}_{x}{ }^{-}$clusters.

To enhance our knowledge for those materials with varying degrees of electron mobility $\left(\mathrm{C}_{60}, \mathrm{CuPC}\right.$ and $\left.\mathrm{Alq}_{3}\right)$, further studies on the fragmentation mechanisms as well as on the ionization processes are required.

\section{References}

[1] W. O. Hofer, in Sputtering by Particle Bombardment III (Eds: R. Behrisch, K. Wittmaack), Springer-Verlag: Berlin Heidelberg, 1991, pp 15.

[2] H. M. Urbassek, W. O. Hofer, in Fundamental Processes in Sputtering of Atoms and Molecules (SPUT92) Book series, P. Sigmund (ed), Kongelige danske videnskabernes selskab: matematisk-Fysiske meddelelser, 1993, 97.

[3] M. L. Yu, in Sputtering by Particle Bombardment III (Eds: R. Behrisch, K. Wittmaack), Springer-Verlag: Berlin Heidelberg, 1991, pp 91.

[4] H. Gnaser, H. Oechsner, Nucl. Instrum. Methods Phys. Res. B 1993, 82, 518.

[5] H. Gnaser, Phys. Rev. B 2001, 63, 45415.

[6] H. Gnaser, Nucl. Instrum. Methods Phys. Res. B 1999, 149, 38.

[7] A. K. Gupta, P. Ayyub, Eur. Phys. J. D 2001, 17, 221.

[8] R. Heinrich, C. Staudt, M. Wahl, A. Wucher, Proceeding of the twelve International Conference on Secondary Ion Mass Spectrometry (SIMS XII) 1999, 111.

[9] H. Yamamoto, F. Esaka, H. Asaoka, Proceeding of the twelve International Conference on Secondary Ion Mass Spectrometry (SIMS XII) 1999, 295.

[10] H. Gnaser, Appl. Surf. Sci. 2003, 203-204, 78.

[11] H. Gnaser, Nucl. Instrum. Methods Phys. Res. B 2000, 164, 705.

[12] R. Golser, H. Gnaser, W. Kutschera, A. Priller, P. Steier, C. Vockenhuber, Nucl. Instrum. Methods Phys. Res. B 2004, 223-224, 221.

[13] E. D. Chambost, B. Boyer, B. Rasser, M. Schuhmacher, Proceeding of the twelve International Conference on Secondary lon Mass Spectrometry (SIMS XII) 1999, 533.

[14] J. Y. Qi, L. Dang, M. D. Chen, W. Wu, Q. E. Zhang, C. T. Au, J. Phys. Chem. A 2008, 112, 12456.

[15] C. G. Zhan, S. Iwata, J. Chem. Phys. 1996, 104, 9058. 